\title{
Prognose de crescimento diamétrico de uma área restaurada no Cerrado
}

\author{
Diametric growth prognosis of a restored area in the Cerrado biome \\ Pronóstico de crecimiento diametral de um área restaurada em el bioma Cerrado
}

Recebido: 10/05/2021 | Revisado: 16/05/2021 | Aceito: 21/05/2021 | Publicado: 07/06/2021

Juliane Cruz Barros

ORCID: https://orcid.org/0000-0001-8772-4551

Universidade Federal de Goiás, Brasil

E-mail: julianecruz98@gmail.com

Hallefy Junio de Souza

ORCID: https://orcid.org/0000-0001-8284-0246

Universidade de Brasília, Brasil

E-mail: hallefyj.souza@gmail.com

Sybelle Barreira

ORCID: https://orcid.org/0000-0003-1482-2411 Universidade Federal de Goiás, Brasil E-mail: sybelleb@ufg.br

\begin{abstract}
Resumo
O Cerrado é o segundo maior bioma da América do Sul ocupando cerca de $23 \%$ do território nacional, mas que vem perdendo grande parte da sua vegetação nativa devido ao desmatamento e a fragmentação antrópica. O estudo teve como objetivo entender o comportamento de uma área restaurada no bioma por meio do prognóstico do fragmento florestal, a partir do método de razão de movimentação das classes. Foram realizados dois inventários florestais, onde foram mensurados todos os indivíduos com CAP (circunferência a altura do peito) superior a $15 \mathrm{~cm}$, para posterior ajuste dos modelos não lineares de incremento periódico, ingresso e mortalidade. Os resultados demonstraram perda da densidade de indivíduos, indicando a passagem de fase do fragmento de "construção" "para "degradação". Os principais fatores da degradação foram, a acessibilidade ao local, descarte de lixo urbano e a falta de planejamento do projeto de restauração, deixando de levar em consideração os princípios de sucessão ecológica e o levantamento das informações de referência do ambiente. Porém, a área apresenta capacidade de regeneração se forem adotadas técnicas que interfiram no processo de restauração, pois houve aumento na taxa do número de indivíduos na menor classe de diâmetro (2023) se comparado aos anos anteriores; aumento do crescimento na penúltima classe, seguido de estabilização; redução da perda de densidade dos indivíduos, entre os intervalos dos anos 2014-2017 e 2017-2023; e tendência de distribuição diamétrica em "J invertido" no ano de 2023.
\end{abstract}

Palavras-chave: Razão de movimentação; Degradação; Sucessão ecológica.

\begin{abstract}
The Cerrado is the second largest biome in South America, occupying about $23 \%$ of the national territory, but which has been losing a large part of its native vegetation due to deforestation and anthropic fragmentation. This study aimed to understand the behavior of an area under restored in the biome, through the prognosis of the forest fragment using the class movement ratio method. Two forest inventories were carried out, in which all individuals with a CAP (circumference at chest height) greater than $15 \mathrm{~cm}$ were measured, for later adjustment of the nonlinear models of periodic increment, entry and mortality. Results showed individuals density loss of, indicating the phase transition from "construction" fragment to "degradation". The main factors of degradation were accessibility to the site, disposal of urban waste and lack of planning for the restoration project, failing to take into account the principles of ecological succession and the survey of environmental reference information. However, the area showed regeneration capacity if the correct techniques are adopted that interfere with the restoration process, as there was an increase in the number of individuals in the smallest diameter class (2023) compared to previous years; increased growth in the penultimate class, followed by stabilization; reduction in the density loss of individuals, between the 2014-2017 and 2017-2023 intervals; and trend of the diametric distribution in "inverted J" in 2023.
\end{abstract}

Keywords: Rate of movement; Degradation; Ecological Succession.

\section{Resumen}

El Cerrado es el segundo bioma más grande de América del Sur, ocupando alrededor del 23\% del territorio nacional, pero que há ido perdiendo gran parte de su vegetación nativa debido a la deforestación antrópica. El studio tuvo como objetivo comprender el comportamiento de um área restaurada em el bioma a través del pronóstico del fragmento de bosque, utilizando el método de razón de movimiento de clases. Se realizaron dos inventarios forestales, donde se midieron todos los individuos com CAP (circunferencia a la altura del pecho) mayor a $15 \mathrm{~cm}$, para posterior ajuste de los modelos no lineales de incremento periódico, ingresso y mortalidad. Los resultados mostraron pérdida de densidad de individuos, lo que indica la transición de fase del fragmento de "construcción" a 
“degradación”. Los principales factores de degradación fueron la accesibilidad al sitio, la disposición de residuos urbanos y la falta de planificación del proyecto de restauración, sin tener em cuenta los principios de sucesión ecológica y el levantamiento de información de referencia sobre el medio ambiente. Sin embargo, el área es capaz de regenerarse si se adoptan técnicas que interfieran com el proceso de restauración, ya que hubo um aumento em el número de individuos em la clase de menor diámentro (2023) en comparación com años anteriores; aumento del crecimiento en la penúltima clase, seguido de estabilización; reducción de la pérdida de densidad de individuos, entre los años 2014-2017 y 2017-2023; y tendencia de distribución diametral em "J invertida" em el año 2023.

Palabras clave: Tasa de movimento; Degradación; Sucesión ecológica.

\section{Introdução}

O Cerrado é o segundo maior bioma da América do Sul (Roitman et al., 2018), ocupando cerca de 23\% do território brasileiro (MapBiomas, 2019), sendo que grande parte já está fragmentada ou foi desmatada, apresentando-se como um grande mosaico de áreas remanescentes.

O conhecimento das fitofisionomias do bioma torna-se fundamental para sua conservação e manejo de suas áreas. O entendimento de como cada fisionomia se comporta em termos de incremento em altura e diâmetro continuam sendo desafios para compreensão quanto ao seu comportamento, evolução, produção e crescimento (Bom, 1996). Cada fragmento tem um incremento específico, variando de região para região, em função da topografia, do clima, dos solos e da sua utilização em períodos anteriores (Fischer, 1987).

O manejo florestal é definido como a administração da floresta para a obtenção de benefícios econômicos, sociais e ambientais (Carvalho, 2019). Por isso, ele é considerado boa alternativa para garantir a continuidade da produção de madeira, sem que haja a alteração de uso do solo nas áreas manejadas (Silva et al., 2014), no entanto, necessita se valer de informações sobre incremento, o crescimento no tempo, qual ciclo de corte entre intervenções, a produtividade, taxa de regeneração, entre outras, para que se estabeleça o manejo sustentável visando ao uso múltiplo e perpetuidade dos seus fragmentos (Mattos et al., 2007).

Segundo Souza et al. (1993) o manejo das florestas nativas passa, obrigatoriamente, pelo conhecimento dos processos de dinâmica de seu crescimento, como também é necessário saber como e quanto as intervenções silviculturais afetam o crescimento das árvores da floresta manejada.

Scolforo et al. (1996) enfatizam que, além de estudos de estrutura e dinâmica das florestas, é importante também conhecer o ciclo de corte e de como o número de arvores por classe de diâmetro evolui ao longo do tempo. Segundo Reis et al. (2018), a sustentabilidade do manejo florestal não é comprometida se a intensidade de corte não ultrapassar a capacidade de recuperação do estoque remanescente em um determinado tempo planejado.

O planejamento do manejo e a conservação desses remanescentes é feito através de estudos de projeção e análises de monitoramento que possibilitem o entendimento dos processos que levam a mudanças na estrutura da floresta (Batista et al., 2016).

A projeção é composta basicamente por modelos que descrevem o crescimento e produção futura de florestas multiâneas por classe de diâmetro, necessariamente analisando todos os aspectos que influenciam a dinâmica populacional em termos de recrutamento, mortalidade, grau de ocupação e competição, sítio e idade, bem como o efeito desses fatores sobre o ingresso en tre as classes diamétricas ao longo da distribuição de frequência da floresta (Nascimento et al., 2012).

A razão de movimentação é um importante modelo utilizado para a prognose em florestas nativas, sendo definida como a proporção de árvores que avançaram no lado direito das classes de diâmetro. Essa metodologia utiliza o incremento diamétrico médio e assume que a distribuição dos diâmetros é uniforme dentro dessas classes (Husch et al., 1982; Scolforo, 1998). 
Sendo assim, o objetivo do trabalho foi avaliar a estrutura diamétrica de uma área restaurada no bioma Cerrado, por meio do seu prognóstico usando o método de razão de movimentação, com a finalidade de subsidiar possíveis intervenções de manejo florestal.

\section{Metodologia}

\section{a) Localização da área de estudo}

O estudo foi realizado na Escola de Agronomia da Universidade Federal de Goiás, Campus Samambaia, com coordenadas $16^{\circ} 36^{\prime} \mathrm{S}, 4^{\circ} 16^{\prime} \mathrm{W}$ e altitude de 727 m, no município de Goiânia (Figura 1).

Figura 1: Mapa de localização da área de estudo, Cinturão Verde.
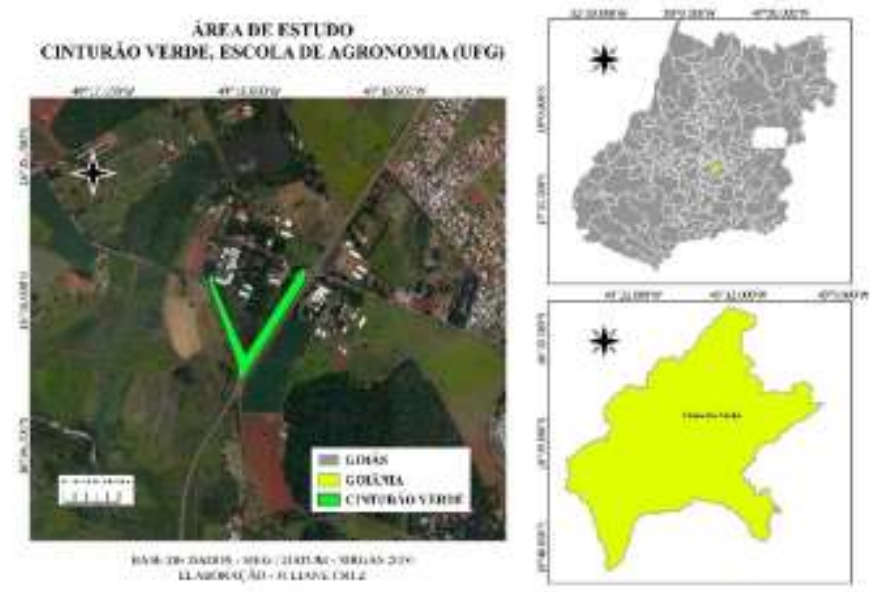

Fonte: Autores (2020).

A área de estudo é denominada de Cinturão Verde com aproximadamente 3,30 ha, e trata-se de um fragmento em processo de restauração com espécies nativas do bioma Cerrado. A implantação desse projeto ocorreu no ano de 2003 , e teve como objetivo principal reconstruir ao máximo as características do ambiente em sua forma original (floresta estacional semidecidual), anteriormente ocupado por um monocultivo de eucalipto.

O clima segundo a classificação de Köpen em maior parte da região é do tipo Aw, caracterizando clima tropical com inverno seco (Beck et al., 2018). A temperatura média é de $23.4^{\circ} \mathrm{C}$, com precipitação anual média equivalente a $1270 \mathrm{~mm}$ (Climate-data, 2021).

\section{b) Coleta de dados}

A coleta quantitativa dos dados (Pereira et al., 2018) seguiu a metodologia descrita por Lopes \& Soares (2013), em que foram realizados dois inventários florestais por meio de Censo 100\% com o intervalo de três anos entre as mensurações, sendo o primeiro em 2014 e o segundo em 2017. Para limite de inclusão dos indivíduos adotamos CAP $\geq 15 \mathrm{~cm}$, todos mensurados com fita métrica.

\section{c) Análise de dados}

Inicialmente para a análise de dados, houve a conversão de CAP para DAP (CAP/ $\pi$ ). Posteriormente, foi determinada a distribuição das classes, através da amplitude dos diâmetros em razão de um número (n) conveniente de intervalos de classe que tivessem a mesma amplitude $(\mathrm{n}=11)$. 
A partir do segundo inventário, foi quantificado o incremento periódico médio por classe de diâmetro através da Equação 1:

$$
\overline{I P D}_{j}=\frac{\sum_{i=1}^{n}\left(D A P_{2 i}-D A P_{1 i}\right)}{N}
$$

em que: $\overline{I P D}_{j}=$ incremento periódico médio em diâmetro da $j$-ésima classe de diâmetro; $D A P_{1 i}=\mathrm{DAP}$ da $i$-ésima árvore na $1^{\circ}$ medição; $D A P_{2 i}=$ DAP da $i$-ésima árvore na $2^{\circ}$ medição; $N=$ número total de árvores em cada classe diamétrica.

A partir do incremento periódico médio e do número de indivíduos ingressantes e mortos por classe diamétrica, realizamos uma pré-seleção de modelos, dos quais, os seguintes modelos não lineares foram selecionados, com base no coeficiente de correlação entre as variáveis estimadas e observadas e erro padrão das estimativas (absoluto e percentual) (Tabela 1):

Tabela 1: Modelos não lineares selecionados para estimar o incremento periódico, ingresso e a mortalidade.

\begin{tabular}{ccc}
\hline Autor & Modelos \\
\hline Logistic & $I P=\frac{\beta_{0}}{\left(1+\beta_{1} * \exp ^{\left(-\beta_{2} * C L D\right)}\right)} \quad(2)$
\end{tabular}

Modified Geometric

Logistic Power

$$
\begin{array}{r}
I=\beta_{0} * C L D^{\frac{\beta_{1}}{C L D}} \\
M=\frac{\beta_{0}}{\left(1+\left(\frac{C L D}{\beta_{1}}\right)^{\beta_{2}}\right)}
\end{array}
$$

$I P=$ incremento periódico por classe de diâmetro; $I=$ ingresso de árvores por classe de diâmetro; $M=$ número de árvores mortas por classe de diâmetro; $C L D=$ valor central da classe de diâmetro.

Fonte: Autores (2020).

Após implementada a movimentação por classe de diâmetro, foram descontadas as árvores mortas e agregadas as árvores que ingressaram em cada classe de diâmetro (Scolforo, 1998).

\section{Resultados e Discussão}

As classes apresentaram amplitude de $5 \mathrm{~cm}$, cujo centro de classe diamétrica inicial foi 7,5 $\mathrm{cm}$ e o centro de classe diamétrica final foi de $57,5 \mathrm{~cm}$, totalizando 11 classes.

Para o ajuste do modelo de incremento periódico foram determinados os seguintes valores de coeficientes e estatísticas de precisão: $\beta_{0}=3,340334 ; \beta_{1}=12,48224$ e $\beta_{2}=0,163406$ com $r=0,93$; Syx $=0,385$ e Syx $\%=14,55$ (Figura 2). 
Figura 2: Incremento periódico por classe de diâmetro.

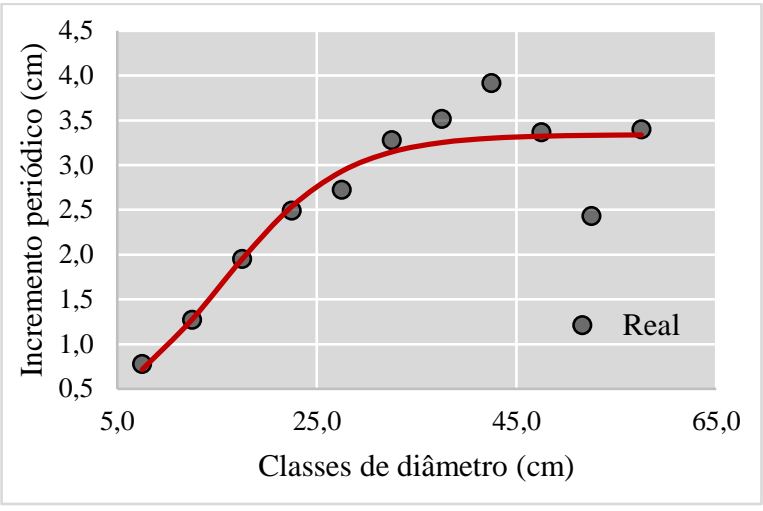

Fonte: Autores (2020).

O incremento periódico, seguido de um leve declínio, ocorreu através do fechamento gradual do dossel nas classes superiores e do aumento da competição por nutrientes entre as plantas (Reis et al., 2014).

Para o ingresso, os coeficientes foram equivalentes a $\beta_{0}=0,025199$ e $\beta_{1}=31,618249$ com $\mathrm{r}=0,99$; Syx $=1,980$ e Syx $\%=15,45$. Enquanto que, para a mortalidade os valores foram $\beta_{0}=121,38 ; \beta_{1}=21,37439$ e $\beta_{2}=6,31135 \mathrm{com} \mathrm{r}=0,99$; Syx $=2,753$ e Syx $\%=7,17$ (Figuras 3 e 4).

Figura 3: Número do ingresso de árvores por classe de diâmetro.

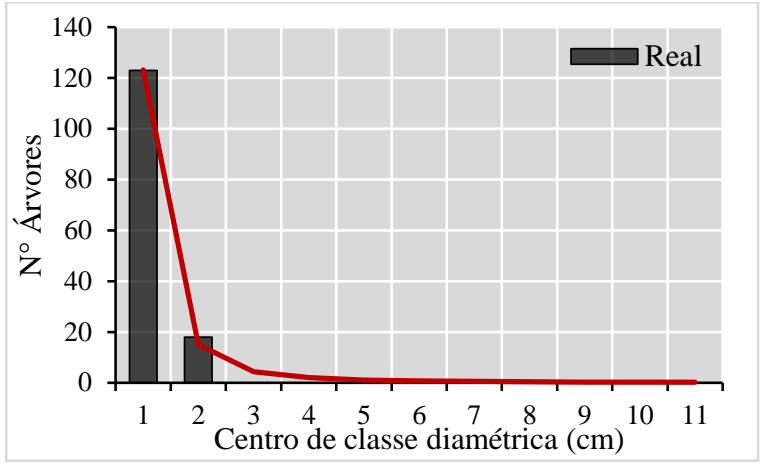

Fonte: Autores (2020).

Figura 4: Número de árvores mortas por classe de diâmetro.

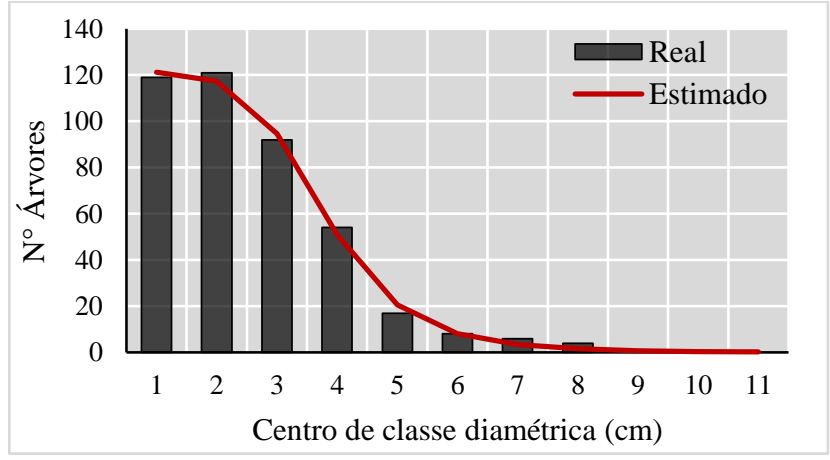

Fonte: Autores (2020).

Os índices de ingresso e mortalidade sugeriram que, na classe de diâmetro 1 , os valores de recrutamento $\left(38,0\right.$ ind.ha $\left.{ }^{-1}\right)$ foram superiores ao de mortalidade (36,2 ind.ha $\mathrm{f}^{-1}$ ), devido a maior abertura do dossel (Dionisio et al., 2018), favorecendo a 
entrada de radiação solar e promovendo a ativação das mudas e do banco de sementes (Reis et al., 2014), logo após, com o fechamento do dossel, a quantidade do número de árvores ingressantes reduziu-se drasticamente (Reis et al., 2019) somado a impossibilidade de novos ingressos nas classes mais avançadas. Por outro lado, a superioridade do índice total de mortalidade $\left(139,7\right.$ ind. $\left.h a^{-1}\right)$ em relação ao índice total de recrutamento $\left(49,4 \mathrm{ind} \cdot h \mathrm{a}^{-1}\right)$, indicou que a floresta está passando por uma fase cíclica (Stepka et al., 2010), no qual, a mortalidade além de ser decorrente de processos naturais de competição, pode ser ocasionada por susceptibilidade à pragas e a doenças (Lisboa et al., 2019). A redução da mortalidade, no entanto, se deu pelo fato do estabelecimento dos indivíduos maduros.

A distribuição dos indivíduos por classes de diâmetro para o ano de 2023 foi calculada em dois intervalos de três anos (2017-2020 e 2020-2023), devido ao tempo decorrente dos inventários realizados. Para todos os anos, houve uma queda na densidade de indivíduos. O número de indivíduos passou de 424,0 ind.h $a^{-1} \mathrm{em} 2017$ para aproximadamente 250,0 ind.ha- $\mathrm{em}^{-1}$ 2023, apontando uma redução de 29,0 ind.ha ${ }^{-1}$.ano ${ }^{-1}$. Semelhante a Fonseca \& Rodrigues (2000), a queda de densidade dos indivíduos, caracterizou a passagem de fase de desenvolvimento do mosaico florestal, de "construção" para "degradação". A fase de construção é caracterizada por uma floresta jovem que amadurece através de um crescimento contínuo de suas árvores constituintes (Whitmore, 1990). Já a fase de degradação é o estágio final da floresta que apresenta árvores com sinais visíveis de degradação ou mortas em pé (Torquebiau, 1986). Porém, se comparado a redução do número de indivíduos entre os anos de

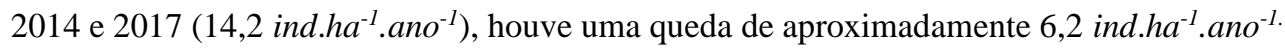

No ano de 2017, cuja a distribuição diamétrica foi a observada, a menor classe de diâmetro $(7,5 \mathrm{~cm})$ apresentou menor número de indivíduos que o centro de classe seguinte $(12,5 \mathrm{~cm}$ ), a taxa de 14,7\%; após três anos (de 2017 para 2020) houve uma redução para 2,5\%. Já em 2023, a quantidade de indivíduos na menor classe de diâmetro $(7,5 \mathrm{~cm})$ conseguiu ser superior à classe seguinte $(12,5 \mathrm{~cm})$, com taxa equivalente a $31,0 \%$.

Em todas as classes houve redução do número de indivíduos com o decorrer dos anos, mas nas classes finais $(52,5 \mathrm{~cm})$ houve um expressivo aumento na quantidade de indivíduos à taxa de aproximadamente 10,7\% ao ano, seguido de estabilização na classe seguinte (Tabela 2).

Tabela 2: Número de indivíduos por hectare nos anos de 2017, 2020 e 2023.

\begin{tabular}{lccc}
\hline \multirow{2}{*}{ CLD } & \multicolumn{3}{c}{ Número de árvores } \\
\cline { 2 - 4 } & 2017 & 2020 & 2023 \\
\hline 7,5 & 325,0 & 263,9 & 240,7 \\
12,5 & 381,0 & 270,8 & 165,9 \\
17,5 & 332,0 & 243,7 & 154,1 \\
22,5 & 192,0 & 158,3 & 118,7 \\
27,5 & 88,0 & 80,9 & 70,7 \\
32,5 & 41,0 & 39,1 & 36,6 \\
37,5 & 20,0 & 19,4 & 18,6 \\
42,5 & 11,0 & 10,7 & 10,4 \\
47,5 & 7,0 & 6,9 & 6,8 \\
52,5 & 1,0 & 1,3 & 1,5 \\
57,5 & 1,0 & 1,0 & 1,0 \\
\hline Total & 1399,0 & 1096,1 & 825,1 \\
\hline Total/ha & 424,0 & 332,1 & 250,0 \\
\hline
\end{tabular}

Fonte: Autores (2020).

Assim, a distribuição dos indivíduos mostrou-se dinâmica, cuja estrutura diamétrica prognosticada se aproximou à estrutura de florestas estacionais semideciduais naturais, mesmo que de forma decrescente, pois mesmo que os índices totais de mortalidade tenham sido superiores aos índices de recrutamento, os resultados não seguiram o mesmo padrão em todas as classes, visto que, na primeira classe de diâmetro com CLD equivalente a 7,5 cm, os valores de recrutamento foram superiores ao de 
mortalidade. Além da queda da mortalidade nas classes respectivas. Contribuindo para a formação da estrutura em "J invertido" no ano de 2023, conforme exibidos nas Figuras 5, 6 e 7.

Figura 5: Distribuição do número de árvores no ano de 2017.

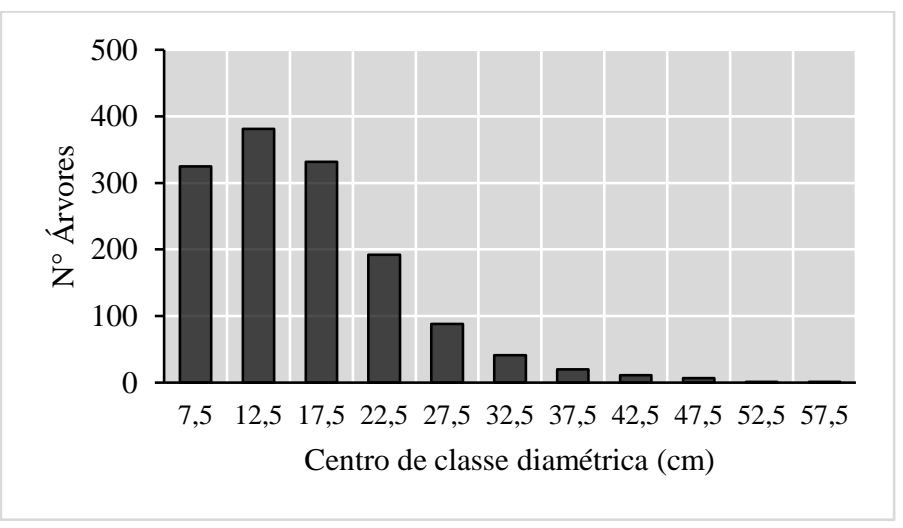

Fonte: Autores (2020).

Figura 6: Distribuição do número de árvores no ano de 2020.

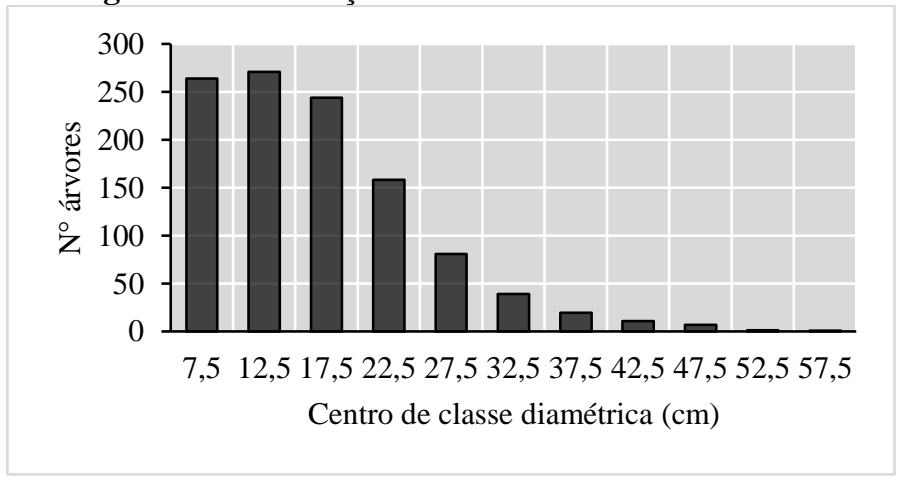

Fonte: Autores (2020).

Figura 7: Distribuição do número de árvores no ano de 2023.

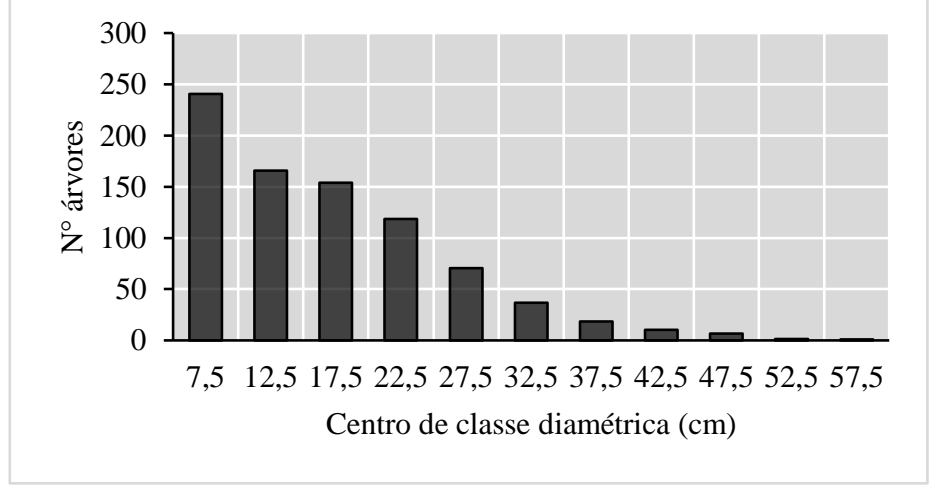

Fonte: Autores (2020).

O comportamento da distribuição do número de indivíduos nas classes diamétricas durante os seis anos, deixou explicito a dificuldade da regeneração natural do ambiente. Dentre os principais motivos da dificuldade da restauração, destacou-se o fator antrópico por meio da circulação de pessoas na área (acessibilidade), o descarte de lixo urbano e a constante limpeza da área, eliminando indivíduos de regeneração natural. Para Lopes et al. (2013), a acessibilidade é o principal fator antrópico modificador da estrutura de um fragmento florestal, além de ser o impacto que apresenta a maior dificuldade de manejo, pois envolve a 
localização geográfica do fragmento, a proximidade a estradas externas e estradas vicinais e a facilidade de visitação. O fator menos relevante é o descarte de lixo urbano, que dentre os impactos causados, há a influência na redução da regeneração natural em locais de maior concentração de lixo (Sobral \& Santana, 2007).

Além do mais, para o processo de recomposição do ambiente semelhante à sua forma original não foram levados em consideração fatores como, a proximidade entre fragmentos de maior área nuclear e proximidade da cobertura vegetal (ecossistema de referência), declividade e erodibilidade do solo (Sartori et al., 2012), assim como, o planejamento de modelos de plantação projetados pela combinação de espaçamento, arranjo e combinação de espécies ou grupos funcionais (Soares et al., 2016). Segundo os padrões internacionais, para prática da restauração ecológica estabelecidos recentemente pela Sociedade Internacional de Resturação Ecológica (Gann et al., 2019), os atributos-chave na caracterização de ecossistemas de referência devem ser: ausência de ameaças externas, composição de espécies, estrutura da comunidade, condições físicas adequadas e funcionamento do ecossistema e trocas externas (fluxos de paisagem).

Dessa forma, a recomposição do ambiente deveria estar baseada em estruturas semelhante à de florestas estacionais semideciduais naturais, levando em consideração a escolha de espécies, conforme o seu crescimento e ocupação do dossel, para consequente desenvolvimento da sucessão natural ecológica.

Assim a adoção da técnica de restauração baseada no ecossistema de referência, aumentaria também, a conectividade funcional do fragmento, uma vez que, segundo (Garcia et al., 2019) a composição e configuração da paisagem afeta diretamente os agentes de dispersão que influi na diversidade genética das população arbóreas, pois a fragmentação não isola reprodutivamente a população amostrada, porque os indivíduos localizados fora da área podem servir como fontes de fluxo gênico entre os fragmentos remanescentes.

Ainda, o percentual de indivíduos encontrados na primeira classe de diâmetro $(29,2 \%)$, foi pouco expressivo se comparado às classes seguintes. Indicando que o fragmento necessita da adoção de técnicas que induzam o seu desenvolvimento, pois se comparando a distribuição diamétrica na primeira classe de diâmetro com a de florestas estacionais semideciduais naturais, os valores são superiores, estando entre 75\% (Bambolim et al., 2018) e 83,4\% (Dias et al., 2019) contidos na mesma classe.

\section{Conclusão}

A projeção da estrutura diamétrica sugere que o fragmento florestal está passando por uma fase cíclica, onde a queda na densidade de indivíduos em todas as classes de diâmetro indica o movimento da fase de construção para a fase de degradação. Os principais fatores que contribuíram para a mudança de fase foram, a acessibilidade, o descarte de lixo urbano e a falta de planejamento na construção do projeto de restauração, deixando assim, de contribuir pra a conectividade funcional do ecossistema. Porém, há a possibilidade de regeneração da área se adotadas técnicas que contribuam para o processo de restauração do ambiente.

Recomenda-se para futuros trabalhos de restauração, o estudo da área do entorno para escolha das espécies a serem implantadas, levando em consideração os princípios de sucessão natural, pautados em parâmetros de crescimento dos indivíduos e ocupação do dossel.

\section{Referências}

Bambolim, A., Donde, A. R., \& Wojciechowsk, J. C. (2018). Análise fitossociológica e estrutura florística de uma floresta estacional decidual. Journal of Neotropical Agriculture, 5(2), 62-68. https://doi.org/10.32404/rean.v5i2.1544

Batista, A. P. B., Rodal, M. J. N., Silva, J. A. A. da, Silva, A. C. B. L. e, Alves Junior, F. T., \& Mello, J. M. de. (2016). Dynamics and prediction of diametric structure in two Atlantic Forest fragments in Northeastern Brazil. Revista Árvore, 40(2), 307-317. https://doi.org/10.1590/0100-67622016000200013

Beck, H. E., Zimmermann, N. E., McVicar, T. R., Vergopolan, N., Berg, A., \& Wood, E. F. (2018). Present and future köppen-geiger climate classification maps 
at 1-km resolution. Scientific Data, 5, 1-12. https://doi.org/10.1038/sdata.2018.214

Bom, R. P. (1996). Proposição de um sistema de manejo para floresta nativa objetivando a sustentabilidade da produção. Curitiba, Brasil: Biblioteca Florestal. Carvalho, A. N. De. (2019). Environmental impacts of community forest management in the tapajós national forest. Agroecossistemas, 11(1), 169-182.

Climate-data. (2021). Goiânia clima. https://pt.climate-data.org/america-do-sul/brasil/goias/goiania-2191/

Dias, P. B., Moreira, L. N., Da Silva, G. F., Pezzopane, J. E. M. E., \& Dias, H. M. H. (2019). Richness, structure and environmental relations in a National Forest in Southeast Brazil. Revista Brasileirade Ciencias Agrarias, 14(4). https://doi.org/10.5039/agraria.v14i4a6897

Dionisio, L. F. S., De Carvalho, J. O. P., Schwartz, G., Leaõ, F. M., \& Castro, T. C. (2018). Post-harvesting increment, recruitment and mortality of Duguetia spp. in the Tapajos National Forest, Pará. Scientia Forestalis/Forest Sciences, 46(119).

Fischer, G. R. (1987). Manejo sustentado de florestas nativas. Joinville, Brasil: Escopa.

Fonseca, R. C. B., \& Rodrigues, R. R. (2000). Structural analysis and aspects of the successional mosaic of a semi-deciduous forest, in Botucatu (São Paulo State, Brazil). Scientia Forestalis/Forest Sciences, 57, 27-43.

Gann, G. D., McDonald, T., Walder, B., Aronson, J., Nelson, C. R., Jonson, J., Hallett, J. G., Eisenberg, C., Guariguata, M. R., Liu, J., Hua, F., Echeverría, C., Gonzales, E., Shaw, N., Decleer, K., Dixon, K. W. (2019). International principles and standards for the practice of ecological restoration. Restoration Ecology, 27(S1), S1-S46.

Gonzales, E., Shaw, N., Decleer, K., \& Dixon, K. W. (2019). International principles and standards for the practice of ecological restoration. Second edition. Restoration Ecology, 27(S1), S1-S46. https://doi.org/10.1111/rec.13035

Garcia, A. S., Bressan, E. A., Ballester, M. V. R., Figueira, A., \& Sebbenn, A. M. (2019). High rates of pollen and seed flow in Hymenaea stigonocarpa on a highly fragmented savanna landscape in Brazil. New Forests, 50(6), 991-1006. https://doi.org/10.1007/s11056-019-09710-3

Husch, B.; Miller, C. I.; Beers, T. W. (1982). Forest Mensuration. New Jersey, Canada: Wiley.

Lisboa, G. D. S., Veres, Q. J. I., Watzlawick, L. F., França, L. C. de J., Cerqueira, C. L., Miranda, D. L. C. de, Stepka, T. F., \& Longhi, R. V. (2019). Fitossociologia e dinâmica de crescimento em um fragmento de Floresta Estacional Semidecidual. Nativa, 7(4), 452. https://doi.org/10.31413/nativa.v7i4.6803

Lopes, S. de F., Júnior, J. A. do P., Vale, V. S. do, \& Schiavini, I. (2013). Impactos ambientais antrópicos como modificadores da estrutura e funcionalidade de florestas estacionais semideciduais no triângulo mineiro, Brasil. Caminhos de Geografia, 14(47), 233-242.

MapBiomas. (2019). Estatísticas de cobertura. https://plataforma.brasil.mapbiomas.org/

Mattos, P. P. De, Taborda, A., Rivera, H., Maria, Y., \& Oliveira, M. De. (2007). Notas Científicas: Crescimento de Araucaria angustifolia na Reserva Florestal Embrapa /Epagri, Caçador, SC. Pesquisa Florestal Brasileira, 55, 107-114.

Nascimento, R. G. M., Machado, S. do A., Figueiredo Filho, A., \& Higuchi, N. (2012). Modelo de projeção por classe diamétrica para florestas nativas: enfoque na função probabilística de Weibull. Pesquisa Florestal Brasileira, 32(70), 93-103. https://doi.org/10.4336/2012.pfb.32.70.93

Pereira, A. S., Shitsuka, D. M., Parreira, F. J., Shitsuka, R. (2018). Metodologia da Pesquisa Científica. Santa Maria, Brasil: UFSM, NTE.

Reis, L. P., de Carvalho, J. O. P., dos Reis, P. C. M., Gomes, J. M., Ruschel, A. R., \& da Silva, M. G. (2014). Crescimento de mudas de Parkia gigantocarpa ducke, em um sistema de enriquecimento em clareiras após a colheita de madeira. Ciencia Florestal, 24(2), 431-436. https://doi.org/10.5902/19805098

Reis, L. P, Souza, A. L. de., Reis, P. C. M. R, Mazzei, L., Binoti, D. H. B. \& Leite, H. G. (2018). Prognose da distribuição diamétrica na Amazônia utilizando redes neurais artificiais e autômatos celulares. Revista Floresta, 48(1), 93-102. doi: http://dx.doi.org/10.5380/rf.v48i1.52748

Reis, L. P., De Souza, A. L., Dos Reis Reis, P. C. M., Mazzei, L., Leite, H. G., Soares, C. P. B., Torres, C. M. M. E., Da Silva, L. F., Ruschel, A. R., \& Rêgo, L. J. S. (2019). Modeling of tree recruitment by artificial neural networks after wood harvesting in a forest in eastern amazon rain forest. Ciencia Florestal, 29(2), 583-594. https://doi.org/10.5902/1980509825808

Sartori, A. A. da C., da Silva, R. F. B., \& Zimback, C. R. L. (2012). Combinação linear ponderada na definição de áreas prioritárias à conectividade entre fragmentos florestais em ambiente sig. Revista Arvore, 36(6), 1079-1090. https://doi.org/10.1590/S0100-67622012000600009

Silva, J. C. da., De Almeida, A. N., \& Pompermaye, R. D. S. (2014). Análise Estratégica do manejo florestal na Amazônia brasileira. Revista Floresta, 44(3), 341. https://doi.org/10.5380/rf.v44i3.33979

Soares, A. A. V., Botelho, S. A., Davide, A. C., \& Faria, J. M. R. (2016). Influence of Ecological Group Composition, Plantation spacing and arrangement in the restoration of riparian forest on reservoir shores. Ciência Florestal, 26(4), 1107. https://doi.org/10.5902/1980509825015

Sobral, I., \& Santana, R. de O. (2007). Avaliação dos impactos ambientais no Parque Nacional Serra de Itabaiana-SE. Caminhos de Geografia, 102-110. http://www.seer.ufu.br/index.php/caminhosdegeografia/article/view/15713

Scolforo, J. R. S.; Pulz, F. A.; Mello, J. M. \& Oliveira-Filho, A. T. (1996). Modelo de produção para floresta nativa como base para manejo sustentado. Revista Cerne, 2(1), 112-137.

Scolforo, J. R. S. (1998). Manejo Florestal. Lavras, Brasil: Editora UFLA.

Souza, A. L.; Araujo, P. A.; Campos, C. C. \& Paula Neto, F. de. (1993). Dinâmica de crescimento em diâmetro de uma floresta primária sem interferência: uma análise pelo tempo de passagem entre classes diamétricas. Revista Árvore, 17(2), 2-16. Retrieved from encurtador.com.br/zHK89 
Research, Society and Development, v. 10, n. 6, e48510615949, 2021

(CC BY 4.0) | ISSN 2525-3409 | DOI: http://dx.doi.org/10.33448/rsd-v10i6.15949

Souza, A. L. de., Soares, C. P. B. Florestas Nativas - Estruturas, Dinâmica e Manejo. Viçosa, Brasil: Editora UFV.

Stepka, T. F., Dias, A. N., Figueiredo Filho, A., Machado, S. do A., \& Sawczuk, A. R. (2010). Prognose da estrutura diamétrica de uma Floresta Ombrófila Mista com os métodos razão de movimentos e matriz de transição. Pesquisa Florestal Brasileira, 30(64), 327-335. https://doi.org/10.4336/2010.pfb.30.64.327

Torquebiau, E. F. (1986). Mosaic patterns in dipterocarp rain forest in Indonesia, and their implications for practical forestry. Journal of Tropical Ecology, 2(4), $301-325$.

Whitmore, T. C. (1990). An introduction to tropical and recruitment in a tropical rain forest. Oxford, United Kingdom: Clarendon Press. 